\title{
Opportunities of information communication technologies for providing pharmaceutical care in the COVID-19 pandemic
}

\author{
Kristina Kilova', Anna Mihaylova ${ }^{2}$, Lily Peikova ${ }^{3}$ \\ 1 Department of Medical Informatics, Biostatistics and e-Learning, Faculty of Public Health, Medical University of Plovdiv, 15-A Vasil Aprilov blvd., \\ Plovdiv, Bulgaria \\ 2 Medical College, Medical University of Plovdiv, 120 Br. Bukstone str., Plovdiv, Bulgaria \\ 3 Department of Pharmaceutical chemistry, Faculty of Pharmacy, Medical University Sofia, Sofia, Bulgaria \\ Corresponding author: Lily Peikova (lily_peikova@yahoo.com)
}

Received 27 July 2020 • Accepted 5 September 2020 • Published 7 January 2021

Citation: Kilova K, Mihaylova A, Peikova L (2021) Opportunities of information communication technologies for providing pharmaceutical care in the COVID-19 pandemic. Pharmacia 68(1): 9-14. https://doi.org/10.3897/pharmacia.68.e56987

\begin{abstract}
This article discusses the opportunities of information and communication technologies for providing pharmaceutical care in the context of the COVID-19 pandemic.

The global COVID-19 pandemic has necessitated a reorientation of the concept of providing health care to large-scale implementation of information technologies to improve the quality of medical and pharmaceutical services for the population. It had a significant impact on pharmacy, which necessitated changes in the management of pharmaceutical care for patients. Rapid and urgent measures were taken by the pharmacists, who found themselves at the forefront. Telepharmacy has proven to be a successful solution in the fight against the virus in the conditions of physical distance. The introduction of modern information and communication technologies worldwide will create significant advantages for more efficient use of resources and management of pharmaceutical care.
\end{abstract}

\section{Keywords}

health care, pharmaceutical services, pharmacist, telemedicine, telepharmacy

\section{Introduction}

The global pandemic of COVID-19 has necessitated a reorientation of the concept of health care provision to large-scale implementation of information technologies to improve the quality of medical and pharmaceutical services for the population. In this situation, the experts of the World Health Organization (WHO) consider as an essential condition the need for up-to-date pharmaceutical information and rapid (operational) provision of procedures for safe and rational use of medicines in the medical practice. One of the possibilities for solving this problem is the remote spread of information among medical professionals and end users of medicines.

In an effort to protect the health of their populations, many governments have imposed blockades, quarantines and restrictions on the movement of people. They fully mobilized healthcare providers at the forefront of the fight against COVID-19 pandemic.

Pharmacists are among the leading health professionals providing basic health services during a pandemic and health crisis. They are highly qualified health professionals licensed to give advice on health issues and medications in order to ensure the rational use of medications by the general public. 
In this pandemic, the role of the pharmacist extends beyond the distribution and supply of medicines. In providing pharmaceutical care during COVID-19, pharmacists focus on identifying, preventing, and resolving drug therapy problems of patients, especially those with chronic diseases (Okoro 2020).

Patients with chronic diseases are among the most at-risk group for infection. This emphasizes the need for pharmaceutical care for this vulnerable group of patients. Some pharmacy services have proven to be effective in optimizing the health outcomes of patients with chronic diseases (Al Raiisi et al. 2019)

These services include the provision of appropriate information on medicinal products, consultations, the supply of permanently available essential medicines for the management of the condition of the chronically ill, the identification and prevention of potential drug-related problems, the follow-up of drug therapy and more (Stemer and Lemmens-Gruber 2011; Al Aqeel et al. 2018).

Despite the key role that pharmacists play in epidemic and pandemic situations, there is a lack of coordinated actions by the governments of different countries in the fight against the virus. Nevertheless, the International Federation of Pharmaceuticals (FIP) has issued guidelines for COVID-19 for pharmacists and pharmacies, providing information on the role and responsibilities of pharmacists working in different settings to limit the spread of the virus (FIP Health Advisory 2020).

According to the recommendations of the WHO Regional Office for Europe (WHO Europe) and the Organization for Economic Co-operation and Development (OECD), many European countries have recently introduced legislative changes to expand the role of pharmacists and alleviate pressure on the rest of the health system (PGEU PR 2020b).

This article discusses the opportunities of information and communication technologies to provide pharmaceutical care in the context of the COVID-19 pandemic.

\section{Materials and methods}

A literature review was carried out through four databases: PubMed, Google Scholar, ScienceDirect and SpringerLink, to identify relevant articles written in English and published until July 10, 2020. Search terms included: COVID-19 and in combination with "pharmaceutical care", telepharmacy" and "pharmacist".

\section{Results and discussion}

\section{Pharmaceutical care}

In modern pharmacy practice the communication with patients is essential. The nature of the work in pharmacies includes not only the preparation and dispensing of medicines, but also interaction with patients and other health professionals in order to provide pharmaceutical care.
According to the Act on Medicinal Products in Human Medicine, the pharmacy is a health institution in which, in addition to all the main activities of storage, preparation, packaging, control and dispensing of drugs under and without a prescription and other products, patients are being consulted and that is known as pharmaceutical care. There are different definitions of the nature of pharmaceutical care, but the most accurate remains the original concept, defined by Michael et al. in 1975: "the care that a patient needs and receives, and which provides him with safe and rational drug use" (Petkova 2017). Later, this definition is expanded to include not only the determination of the necessary drug therapy, but also all the services that the patient needs to ensure the optimal safe and effective therapy (Felix 2004).

The philosophy of pharmaceutical care defines pharmacists as directly responsible for the patients they serve. The purpose of counselling patients is to improve their quality of life and the quality of pharmaceutical care. The presence of drug deficiencies such as adverse drug reactions, drug interactions, errors in the use of drugs, etc., as well as noncompliance with the therapeutic regimen indicates the need for intervention of pharmacists as drug experts (Dimitrova and Petkova 2005).

\section{Telemedicine and telepharmacy}

With the development of information and telecommunication technologies, some specialists consider the provision of remote information for effective management of pharmaceutical care as promising (Clifton et al. 2003; Heaton et al. 2003; Khan 2008; Douglas 2010).

Initially, the term "telepharmacy" was used to refer to the process of remote ordering and dispensing of drugs, and subsequently significantly expanded its scope of activities (Margolis 2008). According to the definition of the WHO and the US Medical Institute: “... telepharmacy is the remote provision of all aspects of pharmaceutical care through telecommunication technologies ..." (Angaran 1999; Friesner and Scott 2009)

Baldoni et al. (2019) define telepharmacy as: “... a form of pharmaceutical care in which pharmacists and patients are not in the same place and can interact using information and communication technology (ICT) tools". Telepharmacy is accepted for the provision of pharmaceutical services in remote areas and coping with the lack of pharmacists.

In the era of general restrictions on face-to-face communication, it would be exemplary for pharmacists to show their empathy, care, and skills to support patients with their medication-related queries remotely. As defined by the American Society of Hospital Pharmacists (ASHP), telepharmacy is the remote provision of pharmacy services by qualified pharmacy personnel (Alexander et al. 2017). As one of the most accessible health care team members, it is expected that the remote counselling services in the pharmacy settings will be expanded significantly (Elnaem and Cheema 2020).

In recent years, significant scientific evidence has been published that remote information provision significant- 
ly improves the quality of pharmaceutical care given in hospital and pharmacy facilities. The Community Health Association of Spokane conducted a large-scale study in 2003 , which substantiated the possibility of access to information on the safe use of drugs through telecommunication channels of representatives of at-risk social and economic groups. As part of the program webcams are installed in the participating pharmacies with the help of which pharmacists conduct remote consultations. The evaluation of the effectiveness showed that over $70 \%$ of the participants were very satisfied with the provided additional service (Clifton et al. 2003).

A study by S. Margolis (2008) found high efficiency of remote counselling and prescription of drugs to patients in settlements with a shortage of pharmacists. In addition, the application of standards helps to minimize errors to a significant extent. In his work H. Hudson (2005) proposes an approach to designing networks that provide medical and pharmaceutical care to patients in sparsely populated areas, which is extremely important in hard-to-reach areas due to lack of infrastructure or adverse weather conditions Furthermore, telepharmacy significantly optimizes the remote interaction between the various structures of health organizations involved in the process of providing and monitoring medicinal products (Bynum et al. 2001; Keeys et al. 2002; Paulino et al. 2004).

A study of the effectiveness of telepharmaceutical services found that with the help of remote consultations from pharmacists it is possible to ensure the simultaneous distribution of drugs and interdisciplinary communication between doctors and pharmacists (Lam and Rose 2009). In addition, there is an opportunity to use telepharmacy as a management tool in connection with minimizing the cost of pharmaceutical services in the hospital sector (Boon 2007).

The results of developments in the field confirm the effectiveness of distance learning for the proper use of asthma inhalers compared to written instructions and the use of telepharmacy as outsourcing of pharmaceutical services (Bynum et al. 2008).

In summary of the presented studies, we found that the effective use of information and communication technologies leads to improving the organization and providing better pharmaceutical care for patients.

\section{Telepharmacy and the provision of pharmaceutical care for patients in the context of the COVID-19 pandemic}

Nowadays, the question that arises is whether telepharmacy can be a tool during the COVID-19 pandemic which can provide health care in the healthcare system. The coronavirus pandemic (COVID-19) has been spreading rapidly around the whole world.

At the same time, telepharmacy is one of the practical aspects of telemedicine, which refers to the provision of pharmaceutical care within the scope of the pharmacist's responsibilities, with a temporal and spatial distance between patients as users of health services and pharmacists as providers of health services (Ameri et al. 2020).
The global pandemic has also had a significant impact on pharmaceutical care, which has necessitated changes in the management of pharmaceutical care for patients. Given the imposed quarantine and recommendations for social isolation, many people avoid visiting even pharmacies because of the fear of COVID-19. This required urgent action by first-line pharmacists. An example of good practice is the application of telepharmacy, the main manifestation of which is the introduction of a number of mobile applications for providing care. In April 2020 a team of German specialists created a free telepharmaceutical portal APOmondo (www.apomondo.de) to provide personal care for patients regardless of geographical distance according to all scientific standards.

After free registration people can contact pharmacies in their area from home and get advice on medicines and medical devices. The provision of information includes advice on efficient and safe medication, advice on self-medication, support on how to use medical devices and aids, etc. An additional terminal with a video function has been created for the needs of patients, as people are looking for direct contact, especially if they are not sure about the correct intake and side effects of the drugs. As in emergencies, incl. coronavirus pandemic, and due to the growing isolation due to digitalization, an essential task of telepharmacy is to provide adequate pharmaceutical care to patients in need. People not only want to be provided with medicines, but also to be cared for and trained when needed, relying on the empathy and professional experience of pharmacists. Similar functions are performed by the portals Apomap, Oddsmaker, 5plus and others. The existence of official structures in the state of emergency will significantly prevent the distribution of counterfeit medicines through the Internet and other illegal providers.

For effective management of remote information by specialists, users and participants through communication channels, it is necessary to monitor the pharmaceutical market, while at the same time carrying out continuous control over the distribution of medicines (Benabdallah et al. 2011).

The pharmaceutical care that pharmacists offer during this pandemic, according to Okoro (2020), includes: continuous provision of medicines and protective equipment; reliability of the provided information; home delivery; telepharmaceutical care.

Despite the disruption of the global supply chain of basic goods due to blockages, pharmacists provide a continuous supply of basic medicines and protective products (hand sanitizers, gloves, face masks, helmets) to meet the needs of patients (Hedima 2020; PGEU 13 March 2020).

The reliability of information is important during a pandemic and health crisis. Pharmacists offer reliable and timely advice to patients, including those who have symptoms of COVID-19, to continue taking all their medications (Okoro 2020; Schiffrin et al. 2020).

Home delivery is aimed at keeping at home patients at increased risk of COVID-19 infection, such as those with chronic diseases. It helps keep patients and customers away from the pharmacy, which could potentially spread 
the virus among pharmacists (Bukhari et al. 2020; Cooper 2020; Koster et al. 2020).

Many pharmacies offer daily delivery of medicines, critical items and dialysis supplies at home in accordance with the international guidelines for pharmacists and pharmacy in the COVID-19 pandemic, in accordance with the recommendations and guidelines for COVID-19 which are specific for each country (FIP 2020; Okoro 2020).

Telepharmaceutical care aims to minimize direct contact between patients and pharmacists. In many developed countries such as the United States, Germany, Spain, Denmark, France, Canada, Italy, Scotland, the Netherlands and others take advantage of the opportunities of information and communication technologies for the delivery of pharmaceutical care through telepharmacy. This includes distance learning, counselling, monitoring and follow-up of drug therapy (Baldoni et al. 2019; Koster et al. 2020; Okoro 2020).

This reduces the risk of infection for both patients and pharmacists. The National United Federation of Pharmacy Owners in Italy reports that as of May 28, there are 15 pharmacists in the country who are victims of COVID-19, who have fulfilled their duty at the forefront of the fight against the disease https://www.federfarma.it/Edicola/ Filodiretto/VediNotizia.aspx?id=21332). The Royal Pharmaceutical Society reported in an article published on June 12, 2020 in The Pharmaceutical Journal that the total number of confirmed deaths from COVID-19 of pharmacists was five (Burns 2020).

Stress and anxiety are normal human reactions during difficult times. The adverse effect of the COVID-19 pandemic has led to improper adherence to drug therapy among patients with chronic diseases and observed self-medication practices. This is a direct consequence of the fact that patients do not receive medical care or do not follow new prescriptions due to fear of infection. Pharmacists are the ones who offer ongoing care to patients, especially those with chronic illnesses, to ensure better adherence to their drug therapies (Koster et al. 2020; Okoro 2020).

The intensive development of information technologies and personal communications creates new challenges for the healthcare system - unlimited access to medical information with increasing requirements for its quality, reliability and security and global coverage of the society through social networks. The digitalization of healthcare is the main direction of its development. The field of distribution of medicines is an integral part of the healthcare system and one of the main factors guaranteeing its stable functioning and accessibility of health services for the population.

Maintaining a permanent communication relationship doctor - patient - pharmacist - supplier of medicinal products is a condition and prerequisite for providing humane, quality and effective health and pharmaceutical care and services. And this requires the urgent introduction of an electronic health record of every citizen / patient / client (of which the medical file is an integral part) (Stoycheva 2012).

One of the tasks of the state policy in the field of distribution of medicines is to raise the awareness among the medical specialists about the problems related to their provision, safe reception, efficiency and quality of the drug therapy. The lack of reliable sources for providing information for both doctors and pharmacists and the public has a negative impact on the effective functioning of the drug system. The exclusion of medical errors in the appointment of drugs creates a solid basis for improving the process of providing pharmaceutical care. Telepharmacy has real potential to contribute to the successful solution of these problems.

Bulgaria falls behind in the development of e-health not only in terms of the health information system, but also in terms of other elements of e-health related to the development of telemedicine, remote monitoring of patients and others. Bulgaria should use the opportunities for telemedicine to address the challenges facing our healthcare system. One of the measures in the project National Health Strategy (2014-2020) is "elaboration and implementation of a concept for the development of telemedicine in the Republic of Bulgaria|. It is necessary to perform an analysis and assessment of the possibility for introduction of telemedicine services in Bulgaria and to elaborate a comprehensive concept for the development of telemedicine in our health system, as well as for telepharmacy.

So far in our country some separate activities and services have been electrified and local registers and databases have been built, but there is no complete software solution and or a unified information system to serve the electronic management of the health sector. The electrification of healthcare in our country will contribute to providing citizens and medical professionals with systematized and summarized medical information, which is essential in the process of diagnosis, determination of therapeutic plan, prescribing a digital prescription, providing treatment and rehabilitation as well as rescuing and saving lives (Galeva 2018).

\section{Conclusion}

The implementation and effective management of innovative information and communication technologies in the activities of pharmacists during a pandemic will provide significant benefits both for achieving goals to improve the quality of pharmaceutical care supply and for even more efficient use of all types of resources in pharmacy. Telepharmacy allows to optimize the activity of pharmacists and to ensure the provision of quality pharmaceutical assistance to the population, especially in the context of the COVID-19 pandemic.

\section{Acknowledgements}

This research was supported by National Scientific Program „Electronic Health in Bulgaria“ (eHealth) D-01200/16.11.2018. 


\section{References}

Al Aqeel S, Abanmy N, AlShaya H (2018) Interventions for improving pharmacist - led patient counselling in the community setting. a systematic review. Systematic Reviews 7(1): 1-13. https://doi. org/10.1186/s13643-018-0727-4

Al Raiisi F, Stewart D, Fernandez-Llimos F, Salgado TM, Mohamed MF, Cunningham S (2019) Clinical pharmacy practice in the care of Chronic Kidney Disease patients: a systematic review. International Journal of Clinical Pharmacy 41(3): 630-666. https://doi. org/10.1007/s11096-019-00816-4

Alexander E, Butler CD, Darr A (2017) ASHP statement on telepharmacy. American Journal of Health-System Pharmacy 74(9): e236-e241. https://doi.org/10.2146/ajhp170039

Ameri A, Salmanizadeh F, Bahaadinbeigy K (2020) Tele-pharmacy: A new opportunity for consultation during the COVID-19 pandemic. Health Policy and Technology 9(3): 281-282. https://doi. org/10.1016/j.hlpt.2020.06.005

Angaran DM (1999) Telemedicine and telepharmacy: current status and future implications. Telemedicine and telepharmacy: current status and future implications. American Journal of Health-System Pharmacy 56(14): 1405-1426. https://doi.org/10.1093/ajhp/56.14.1405

Baldoni S, Amenta F, Ricci G (2019) Telepharmacy services: present status and future perspectives: a review. Medicina 55: 1-12. https://doi. org/10.3390/medicina55070327

Benabdallah G, Benkirane R, Khattabi A, Edwards IR, Bencheikh RS (2011) The involvement of Pharmacovigilance Centres in medication errors detection: a questionnaire-based analysis. International Journal of Risk \& Safety in Medicine 23(1): 17-29. https://doi.org/10.3233/JRS-2011-0513

Boon AD (2007) Telepharmacy at a critical access hospital. American Journal of Health-System Pharmacy 64(3):242-244. https://doi. org/10.2146/ajhp060392

Bukhari N, Rasheed R, Nayyer B (2020) Pharmacists at the frontline beating the COVID-19 pandemic. Journal of Pharmaceutical Policy and Practice 13: 1-4. https://doi.org/10.1186/s40545-020-00210-w

Burns C (2020) Pharmacist dies from COVID-19, bringing total number of confirmed pharmacy staff deaths to five. The Pharmaceutical Journal 304, No 7938. https://doi.org/10.1211/PJ.2020.20208063

Bynum A, Hopkins D, Thomas A, Copeland N, Irwin C (2001) The effect of telepharmacy counselling on metered-dose inhaler technique among adolescents with asthma in rural Arkansas. Telemedicine Journal and e-Health 7(3): 207-217. https://doi. org/10.1089/153056201316970902

Clifton GD, Byer H, Heaton K, Haberman DJ, Gill H (2003) Provision of pharmacy services to underserved populations via remote dispensing and two-way videoconferencing. American Journal of Health-System Pharmacy 60(24): 2577-2582. https://doi. org/10.1093/ajhp/60.24.2577

Cooper R (2020) Community urged to avoid stockpiling medicines. https://www.australianpharmacist.com.au/community-urged-to-avoid-stockpiling-medicines/?doing_wp_cron=159450 2836.8849840164184570312500

Galeva S, Danova N, Grigorov E (2018) Electronisation of Healthcare in Bulgaria. Yearbook of Hospital Pharmacy 4(1): 24-32. https://doi. org/10.14748/.v4i1.5511

Dimitrova Z, Petkova V (2005) Pharmaceutical Care. University press Sv. Kliment Ohridski, Sofia, 15 pp.
Douglas S, Wakefield D (2010) Implementation of a telepharmacy service to provide round-the-clock medication order review by pharmacists. American Journal of Health-System Pharmacy 67(23): 20522057. https://doi.org/10.2146/ajhp090643

Elnaem MH, Cheema E (2020) Caring for patients with diabetes during COVID-19 pandemic: Important considerations for pharmacists [published online ahead of print, 2020 May 31]. Research in Social and Administrative Pharmacy S1551-7411(20): 1-4. https://doi. org/10.1016/j.sapharm.2020.05.030

Felix S (2004) CCRA recognizes pharmacy services. Pharm Post 12: 1-6. Friesner D, Scott DM (2009) Exploring the formation of patient satisfaction in rural community telepharmacies. Journal of the American Pharmacists Association 49(4): 509-518. https://doi.org/10.1331/ JAPhA.2009.08110

FIP Health Advisory (2020) COVID-19 Pandemic: Guidelines for Pharmacists and the Pharmacy Workforce March 26. 1-29. https://www. fip.org/files/content/priority-areas/coronavirus/COVID-19-Guidelines-for-pharmacists-and-the-pharmacy-workforce.pdf

Hedima EW, Adeyemi MS, Ikunaiye NY (2020) Community Pharmacists: On the frontline of health service against COVID-19 in LMICs [published online ahead of print, 2020 Apr 17]. Research in Social and Administrative Pharmacy S1551-7411(20). https://doi. org/10.1016/j.sapharm.2020.04.013

Hudson HE (2005) Rural telemedicine: lessons from Alaska for developing regions. Telemedicine and e-Health 11(4): 460-467. https://doi. org/10.1089/tmj.2005.11.460

Keeys CA, Dandurand K, Harris J, Gbadamosi L, Vincent J, Jackson-Tyger B, King J (2002) Providing nighttime pharmaceutical services through telepharmacy. American Journal of Health-System Pharmacy 59(8): 716-721. https://doi.org/10.1093/ajhp/59.8.716

Khan S, Snyder HW, Rathke AM, Scott DM, Peterson CD (2008) Is there a successful business case for telepharmacy? Telemedicine and e-Health 14(3): 235-244. https://doi.org/10.1089/tmj.2007.0045

Koster ES, Philbert D, Bouvy ML (2020) Impact of the COVID-19 epidemic on the provision of pharmaceutical care in community pharmacies. Research in Social and Administrative Pharmacy S15517411: 1-3. https://doi.org/10.1016/j.sapharm.2020.07.001

Lam AY, Rose D (2009) Telepharmacy services in an urban community health clinic system. Journal of the American Pharmacists Association 49(5): 652-659. https://doi.org/10.1331/JAPhA.2009.08128

Margolis SA, Ypinazar VA (2008) Tele-pharmacy in remote medical practice: the Royal Flying Doctor Service Medical Chest Program. Rural Remote Health 8(2): e937.

National Health Strategy (2014-2020) National Health Strategy. https:// www.mh.government.bg/media/filer_public/2015/06/26/proekt-nacionalna-zdravna-strategiq-2014-2020.pdf

Okoro R (2020) COVID-19 pandemic: The role of community pharmacists in chronic kidney disease management supportive care. Research in Social and Administrative Pharmacy. https://doi. org/10.1016/j.sapharm.2020.07.008

Paulino EI, Bouvy ML, Gastelurrutia MA, Guerreiro M, Buurma H, ESCP-SIR Rejkjavik Community Pharmacy Research Group (2004) Drug related problems identified by European community pharmacists in patients discharged from hospital. Pharmacy World \& Science 26(6): 353-360. 
Petkova V (2017) Pharmaceutical care - contemporary approach for patients therapy optimization. Social medicine 2-3: 3-6.

PGEU PR (2020a) PGEU PR Community pharmacists in the frontline in the fight against the novel corona-virus disease (COVID-19). [13 March 2020] https://www.pgeu.eu/wp-content/uploads/2020/03/PR-Community-Pharmacists-in-the-frontline-in-the-fight-against-coronavirus.pdf PGEU PR (2020b) PGEU PR Community pharmacists supporting patients and healthcare systems during COVID-19 [15 May 2020] https://www.pgeu.eu/wp-content/uploads/2020/05/PGEU-PRCommunity-Pharmacists-supporting-patients-and-healthcare-systems-during-COVID-19-15-May.pdf
Schiffrin E, Flack J, Ito S, Muntner P, Webb R (2020) Hypertension and COVID-19. American Journal of Hypertension 33(5): 373-374. https://doi.org/10.1093/ajh/hpaa057

Stemer G, Lemmens-Gruber R (2011) Clinical pharmacy activities in chronic kidney disease and end-stage renal disease patients: a systematic literature review. BMC Nephrology volume 12: 35. https:// doi.org/10.1186/1471-2369-12-35

Stoycheva M (2012) The patient / client and pharmaceutical care and services in the competitive market environment of the drug supply. Scientific works of the University of Ruse 51(8.3): 155-160. 